\title{
frailtyHL: A Package for Fitting Frailty Models with $\mathrm{H}$-likelihood
}

\author{
by Il Do Ha, Maengseok Noh and Youngjo Lee
}

\begin{abstract}
We present the frailtyHL package for fitting semi-parametric frailty models using hlikelihood. This package allows lognormal or gamma frailties for random-effect distribution, and it fits shared or multilevel frailty models for correlated survival data. Functions are provided to format and summarize the frailtyHL results. The estimates of fixed effects and frailty parameters and their standard errors are calculated. We illustrate the use of our package with three wellknown data sets and compare our results with various alternative R-procedures.
\end{abstract}

\section{Introduction}

Frailty models with a non-parametric baseline hazard have been widely adopted for the analysis of survival data (Hougaard, 2000; Duchateau and Janssen, 2008). The frailtyHL package (Ha et al., 2012) implements h-likelihood procedures (Ha et al., 2001; Ha and Lee, 2003, 2005) for frailty models. The package fits Cox proportional hazards models (PHMs) with random effects, or frailties. The lognormal or gamma distribution can be adopted as the frailty distribution, corresponding to the normal or log-gamma distribution for the log frailties. H-likelihood obviates the need for marginalization over the frailty distribution, thereby providing a statistically efficient procedure for various random-effect models (Lee et al., 2006).

The main function is frailtyHL (). For instance,

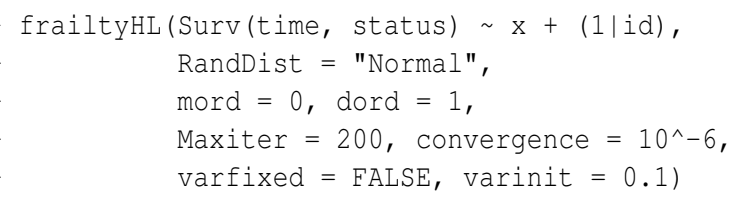

fits a lognormal frailty model. The first argument is a formula object, with the response on the left of a operator, and the terms for the fixed and random effects on the right. The response is a survival object as returned by the Surv function (Therneau, 2011). Here, time and status denote survival time and censoring indicator taking value 1 or 0 for uncensored or censored observations, respectively; $\mathrm{x}$ denotes a fixed covariate and id denotes the subject identifier. The expression ( $1 \mid i d)$ specifies a random intercept model ( $(x \mid$ id) would specify a random intercept, random slope model). The parameters mord and dord are the orders of Laplace approximations to fit the mean parameters (mord $=0$ or 1 ) and the dispersion parameters (dord $=1$ or 2 ), respectively. The
Maxiter parameter specifies the maximum number of iterations and convergence specifies the tolerance of the convergence criterion. If varfixed is specified as TRUE (or FALSE), the value of one or more of the variance terms for the frailties is fixed (or estimated) with starting value (e.g. 0.1$)$ given by varinit.

Previously, frailty models have been implemented in several $\mathrm{R}$ functions such as the coxph () function in the survival package (Therneau, 2010) and the coxme () function in the coxme package (Therneau, 2011), based on penalized partial likelihood (PPL), the phmm() function in the phmm package (Donohue and $\mathrm{Xu}, 2012$ ), based on a Monte Carlo EM (MCEM) method, and the frailtyPenal () function in the frailtypack package (Gonzalez et al., 2012), based on penalized marginal likelihood. The phmm package fits one-component frailty models, although it does allow multivariate frailties. The coxme () function can fit the multi-component model as shown in Example 2. Results from frailtyHL are compared with those from survival, coxme and phmm.

Recently, the h-likelihood procedures of Lee and Nelder (1996) for fitting hierarchical generalized linear models (HGLMs) have been implemented using the $h g \operatorname{lm}($ ) function in the hglm package (Alam et al., 2010), the HGLMfit() function in the HGLMMM package (Molas, 2010) and the dhglmfit () function in the dhglm package (Noh and Lee, 2011). The frailtyHL package is for fitting frailty models with a non-parametric baseline hazard, providing estimates of fixed effects, random effects, and variance components as well as their standard errors. In addition, it provides a statistical test for the variance components of frailties and also three AIC criteria for the model selection.

\section{Frailty models}

The frailtyHL package makes it possible to

1. fit models with log-normal and gamma frailty distributions and

2. estimate variance components when the frailty structure is shared or nested.

For illustration, we present two models below and show how to fit these models using frailtyHL () in the Examples section.

\section{One-component frailty models}

Suppose that data consist of right censored time-toevent observations from $q$ subjects (or clusters), with 
$n_{i}$ observations each; $i=1, \ldots, q$. Let $T_{i j}$ be the survival time for the $j$ th observation of the $i$ th subject; $j=1, \ldots, n_{i}$. Here, $n=\sum_{i} n_{i}$ is the total sample size and $n_{i}$ is the cluster size. Let $C_{i j}$ be the corresponding censoring time and let $y_{i j}=\min \left\{T_{i j}, C_{i j}\right\}$ and $\delta_{i j}=I\left(T_{i j} \leq C_{i j}\right)$ be the observable random variables, where $I(\cdot)$ is the indicator function.

Given the common unobserved frailty for the $i$ th subject $u_{i}$, the conditional hazard function of $T_{i j}$ is of the form

$$
\lambda_{i j}\left(t \mid u_{i}\right)=\lambda_{0}(t) \exp \left(x_{i j}^{T} \beta\right) u_{i}
$$

where $\lambda_{0}(\cdot)$ is an unspecified baseline hazard function and $\beta=\left(\beta_{1}, \ldots, \beta_{p}\right)^{T}$ is a vector of regression parameters for the fixed covariates $x_{i j}$. Here, the term $x_{i j}^{T} \beta$ does not include an intercept term because of identifiability. Assume that the frailties $u_{i}$ are independent and identically distributed (i.i.d) random variables with a frailty parameter $\alpha$; the gamma and lognormal frailty models assume gamma and lognormal distributions for $u_{i}$, respectively. That is, our package allows

- gamma frailty with $E\left(u_{i}\right)=1$ and $\operatorname{var}\left(u_{i}\right)=\alpha$, and

- lognormal frailty having $v_{i}=\log u_{i} \sim N(0, \alpha)$.

The model represented by (1) is known as a shared or one-component frailty model (Hougaard, 2000).

\section{Multi-component frailty models}

We can fit a multi-component model as below (Ha et al., 2007):

$$
X \beta+Z^{(1)} v^{(1)}+Z^{(2)} v^{(2)}+\cdots+Z^{(k)} v^{(k)},
$$

$X$ is the $n \times p$ model matrix, $Z^{(r)}(r=1,2, \ldots, k)$ are the $n \times q_{r}$ model matrices corresponding to the $q_{r} \times 1$ frailties $v^{(r)}$, and $v^{(r)}$ and $v^{(l)}$ are independent for $r \neq l$.

For example, the CGD data (Fleming and Harrington, 1991) have a multilevel structure in which patients nested within centers have recurrent infection times.

Later, we analyze these data using model (2) with $k=2$. Here, the frailty structures are:

$$
\begin{gathered}
v^{(1)}: \text { center frailty } \sim N\left(0, \alpha_{1} A_{1}\right), \\
v^{(2)}: \text { patient frailty } \sim N\left(0, \alpha_{2} A_{2}\right),
\end{gathered}
$$

where $A_{r}=I_{r}(r=1,2)$ are the $q_{r} \times q_{r}$ identity matrices, and $q_{1}$ and $q_{2}$ are the number of centers and patients, respectively. Notice that the corresponding $Z^{(1)}$ to $v^{(1)}$ (or $Z^{(2)}$ to $v^{(2)}$ ) is, respectively, a matrix of indicator variables such that $Z_{s t}^{(1)}=1$ (or $Z_{s t}^{(2)}=1$ ) if observation $s$ is a member of center (or patient) $t$ and 0 otherwise (Therneau and Grambsch, 2000). This is called the multilevel frailty model (Yau, 2001; Ha et al., 2007).

\section{H-likelihood theory}

The h-likelihood $h$ (Ha et al., 2001) for frailty model (1) is defined by

$$
h=h\left(\beta, \lambda_{0}, \alpha\right)=\ell_{0}+\ell_{1}
$$

where

$$
\begin{aligned}
\ell_{0} & =\sum_{i j} \log f\left(y_{i j}, \delta_{i j} \mid u_{i} ; \beta, \lambda_{0}\right) \\
& =\sum_{i j} \delta_{i j}\left\{\log \lambda_{0}\left(y_{i j}\right)+\eta_{i j}\right\}-\sum_{i j} \Lambda_{0}\left(y_{i j}\right) \exp \left(\eta_{i j}\right)
\end{aligned}
$$

is the sum of conditional log densities for $y_{i j}$ and $\delta_{i j}$ given $u_{i}$, and

$$
\ell_{1}=\sum_{i} \log f\left(v_{i} ; \alpha\right)
$$

is the sum of $\log$ densities for $v_{i}=\log u_{i}$ with parameter $\alpha$. Here, $\eta_{i j}=x_{i j}^{T} \beta+v_{i}$ is the linear predictor for the hazards, and

$$
\Lambda_{0}(t)=\int_{0}^{t} \lambda_{0}(k) d k
$$

is the baseline cumulative hazard function.

The functional form of $\lambda_{0}(t)$ in (1) is unknown; following Breslow (1972), we consider $\Lambda_{0}(t)$ to be a step function with jumps at the observed event times:

$$
\Lambda_{0}(t)=\sum_{k: y_{(k)} \leq t} \lambda_{0 k}
$$

where $y_{(k)}$ is the $k$ th $(k=1, \ldots, l)$ smallest distinct event time among the $y_{i j}{ }^{\prime}$ s, and $\lambda_{0 k}=\lambda_{0}\left(y_{(k)}\right)$. However, the dimension of $\lambda_{0}=\left(\lambda_{01}, \ldots, \lambda_{0 l}\right)^{T}$ increases with the sample size $n$. For inference, Ha et al. (2001) proposed the use of the profile h-likelihood with $\lambda_{0}$ eliminated, $\left.h^{*} \equiv h\right|_{\lambda_{0}=\widehat{\lambda}_{0}}$, given by

$$
h^{*}=h^{*}(\beta, \alpha)=\ell_{0}^{*}+\ell_{1},
$$

where

$$
\begin{aligned}
\ell_{0}^{*} & =\sum_{i j} \log f^{*}\left(y_{i j}, \delta_{i j} \mid u_{i} ; \beta\right) \\
& =\sum_{i j} f\left(y_{i j}, \delta_{i j} \mid u_{i} ; \beta, \widehat{\lambda}_{0}\right)
\end{aligned}
$$

does not depend on $\lambda_{0}$, and

$$
\widehat{\lambda}_{0 k}(\beta, v)=\frac{d_{(k)}}{\sum_{(i, j) \in R_{(k)}} \exp \left(\eta_{i j}\right)},
$$

are solutions of the estimating equations, $\partial h / \partial \lambda_{0 k}=$ 0 , for $k=1, \ldots, l$. Here, $d_{(k)}$ is the number of events at $y_{(k)}$ and

$$
R_{(k)}=R\left(y_{(k)}\right)=\left\{(i, j): y_{i j} \geq y_{(k)}\right\}
$$


is the risk set at $y_{(k)}$. Therneau and Grambsch (2000) and Ripatti and Palmgren (2000) proposed an h-likelihood (3), while using the partial likelihood (Breslow, 1974) for $\ell_{0}$. They call it the penalized partial likelihood (PPL) $h_{p}$, defined by

$$
\begin{aligned}
h_{p}(\beta, v, \alpha)= & \sum_{i j} \delta_{i j} \eta_{i j}- \\
& \sum_{k} d_{(k)} \log \left\{\sum_{i j \in R_{(k)}} \exp \left(\eta_{i j}\right)\right\}+\ell_{1} .
\end{aligned}
$$

Furthermore, Ha et al. (2001) and Ha et al. (2010) have shown that $h^{*}$ is proportional to the PPL $h_{p}$ because

$$
\begin{aligned}
h^{*} & =\sum_{k} d_{(k)} \log \widehat{\lambda}_{0 k}+\sum_{i j} \delta_{i j} \eta_{i j}-\sum_{k} d_{(k)}+\ell_{1} \\
& =h_{p}+\sum_{k} d_{(k)}\left\{\log d_{(k)}-1\right\}
\end{aligned}
$$

where $\sum_{k} d_{(k)}\left\{\log d_{(k)}-1\right\}$ is a constant that does not depend upon unknown parameters. Notice here that PPL $h_{p}$ does not depend on nuisance parameters $\lambda_{0}$. Thus, Lee and Nelder's $(1996 ; 2001)$ h-likelihood procedure for HGLMs can be directly used to fit frailty models based on $h_{p}$ (Ha et al., 2010).

\section{Review of estimation procedures}

Lee and Nelder $(1996,2001)$ have proposed the use of the Laplace approximation based on the h-likelihood when the marginal likelihood, $m=\log \left\{\int \exp (h) d v\right\}$, is hard to obtain. To reduce the bias in small cluster sizes higher-order approximations for the mean $(\beta)$ and the frailty variance $(\alpha)$ have been developed. The lower-order approximation is computationally efficient, but could have large biases when cluster sizes are small (Ha and Lee, 2003, 2005; Ha et al., 2010).

The h-likelihood procedures use the Breslow method for handling tied event times, while the PPL procedures allow the Efron method. For estimating $\beta$, the h-likelihood methods allow the Laplace approximation $p_{v}\left(h_{p}\right)$ to a marginal partial likelihood $m_{p}=\log \left\{\int \exp \left(h_{p}\right) d v\right\}$, but the PPL procedures do not. For estimating $\alpha$, the PPL methods use adjusted profile h-likelihoods $p_{v}\left(h_{p}\right)$ and $p_{\beta, v}\left(h_{p}\right)$ which give maximum partial likelihood (MPL) and restricted maximum partial likelihood (REMPL) estimators, respectively. In contrast, the h-likelihood method uses the restricted partial likelihood (based upon the firstorder Laplace approximation $p_{\beta, v}\left(h_{p}\right)$ or the secondorder Laplace approximation $\left.s_{\beta, v}\left(h_{p}\right)\right)$ for REMPL estimators. Here $p_{v}\left(h_{p}\right)$ and $p_{\beta, v}\left(h_{p}\right)$ are defined as follows:

$$
p_{v}\left(h_{p}\right)=\left.\left[h_{p}-\frac{1}{2} \log \operatorname{det}\left\{D\left(h_{p}, v\right) /(2 \pi)\right\}\right]\right|_{v=\hat{v}}
$$

where $D\left(h_{p}, v\right)=-\partial^{2} h_{p} / \partial v^{2}$ and $\hat{v}$ solves $\partial h_{p} / \partial v=$ 0 , which is the first-order Laplace approximation of $m_{p}$, and

$p_{\beta, v}\left(h_{p}\right)=\left.\left[h_{p}-\frac{1}{2} \log \operatorname{det}\left\{\frac{D\left(h_{p},(\beta, v)\right)}{2 \pi}\right\}\right]\right|_{\beta=\hat{\beta}, v=\hat{v},}$ where $D\left(h_{p},(\beta, v)\right)=-\partial^{2} h_{p} / \partial(\beta, v)^{2}$ and $(\hat{\beta}, \hat{v})$ solves $\partial h_{p} / \partial(\beta, v)=0$, which becomes the Cox and Reid (1987) adjusted profile marginal likelihood, eliminating fixed effects $\beta$ by conditioning their asymptotic sufficient statistics $\hat{\beta}$, in addition to eliminating random effects $v$ by the first-order Laplace approximation (Ha and Lee, 2003; Lee et al., 2006). The corresponding second-order approximation is

$$
s_{\beta, v}\left(h_{p}\right)=p_{\beta, v}\left(h_{p}\right)-\left\{F\left(h_{p}\right) / 24\right\},
$$

with

$$
\begin{aligned}
F\left(h_{p}\right)=\operatorname{tr}\left[-\left\{3 \frac{\partial^{4} h_{p}}{\partial v^{4}}+5 \frac{\partial^{3} h_{p}}{\partial v^{3}} D\left(h_{p}, v\right)^{-1} \frac{\partial^{3} h_{p}}{\partial v^{3}}\right\}\right. \\
\left.\times D\left(h_{p}, v\right)^{-2}\right]\left.\right|_{v=\widehat{v} .}
\end{aligned}
$$

\begin{tabular}{|c|c|c|c|}
\hline \multirow[b]{2}{*}{ Method } & \multicolumn{2}{|c|}{ Criterion } & \multirow[t]{2}{*}{ Literature } \\
\hline & $\beta$ & $\alpha$ & \\
\hline $\mathrm{HL}(0,1)$ & $h_{p}$ & $p_{\beta, v}\left(h_{p}\right)$ & Ha and Lee (2003) \\
\hline $\mathrm{HL}(0,2)$ & $h_{p}$ & $s_{\beta, v}\left(h_{p}\right)$ & Ha and Lee (2003) \\
\hline $\mathrm{HL}(1,1)$ & $p_{v}\left(h_{p}\right)$ & $p_{\beta, v}\left(h_{p}\right)$ & Ha et al. (2012) \\
\hline $\mathrm{HL}(1,2)$ & $p_{v}\left(h_{p}\right)$ & $s_{\beta, v}\left(h_{p}\right)$ & Ha et al. (2012) \\
\hline coxph () & $h_{p}$ & $p_{\beta, v}\left(h_{p}\right)$ & $\begin{array}{l}\text { Therneau (2010) } \\
\text { for LN FM }\end{array}$ \\
\hline coxph () & $h_{p}$ & $m$ & $\begin{array}{l}\text { Therneau (2010) } \\
\text { for gamma FM }\end{array}$ \\
\hline coxme () & $h_{p}$ & $p_{v}\left(h_{p}\right)$ & $\begin{array}{l}\text { Therneau (2011) } \\
\text { for LN FM }\end{array}$ \\
\hline phmm () & $m$ & $m$ & $\begin{array}{l}\text { Donohue and } \\
\text { Xu (2012) } \\
\text { for LN FM }\end{array}$ \\
\hline
\end{tabular}

To reduce the computational burden Ha et al. (2010) used $F(h)$ instead of $F\left(h_{p}\right)$.

Table 1: Estimation criteria for h-likelihood (HL(mord, dord)), PPL (coxph(), coxme()) and marginal likelihood ( phmm()) for lognormal (LN) and gamma frailty models (FM)

Table 1 shows historical developments of estimating criteria for frailty models. The frailtyHL () function provides estimators based on the h-likelihood. As the orders in mord and dord increase, the bias of estimator is reduced, but the calculation becomes computationally intensive due to the extra terms. 
We recommend the use of $\operatorname{HL}(1,1)$ for the lognormal frailty and of $\operatorname{HL}(1,2)$ for the gamma frailty. However, $\operatorname{HL}(0,1)$ for the lognormal frailty and $\operatorname{HL}(0,2)$ for the gamma frailty often perform well if $\alpha$ is not large. Note that the variance matrices of $\hat{\tau}=(\hat{\beta}, \hat{v})$ and $\hat{\alpha}$ are directly obtained from the Hessian matrices, $\left\{-\partial^{2} h_{p} / \partial \tau^{2}\right\}^{-1}$ and $\left\{-\partial^{2} p_{\beta, v}\left(h_{p}\right) / \partial \alpha^{2}\right\}^{-1}$, respectively; the frailtyHL package provides the standard errors (SEs) of $\hat{\alpha}$ as well as $\hat{\beta}$. For the use of standard errors of $\hat{v}-v$, see Lee and Ha (2010), Lee et al (2011) and Ha et al. (2011).

Based on the PPL methods, the coxph() and coxme () functions, respectively, implement REMPL and MPL estimators for $\alpha$ in the lognormal frailty model, and the coxph () function the maximum likelihood (ML) estimators, maximizing the marginal likelihood $m$, for $\alpha$ in the gamma frailty model. For comparison, we present the Breslow and Efron methods for handling ties in survival times in the coxph () and coxme () functions; Therneau (2010) recommended the Efron method. For the lognormal frailty the ML estimator maximizing $m$ is available via the phmm () function, but care must be taken to ensure that the MCEM algorithm has converged (Donohue and $\mathrm{Xu}, 2012)$. However, the ML estimator can be biased when the number of nuisance parameters increases with the sample size (Ha et al., 2010).

Furthermore, for the lognormal frailty the coxph () function uses the existing codes in linear mixed models so that it misses the $\partial \hat{v} / \partial \alpha$ term in solving the score equation $\partial p_{\beta, v}\left(h_{p}\right) / \partial \alpha=0$; this can lead to an underestimation of the parameters, especially when the number of subjects $q$ is large or censoring is high (Lee et al., 2006; Ha et al., 2010). To overcome this problem, in gamma frailty models Therneau and Grambsch (2000) develop the code for the ML estimator for $\alpha$.

\section{Fitting algorithm}

Suppose that $\operatorname{HL}(0,1)$ is used. The fitting algorithm is as follows:

Step 1: Take $(0,0,0.1)$ as initial estimates of components of $(\beta, v, \alpha)$.

Step 2: Given $\hat{\alpha}$, new estimates $(\hat{\beta}, \hat{v})$ are obtained by solving the joint estimating equations $\partial h_{p} / \partial(\beta, v)=\left.\{\partial h / \partial(\beta, v)\}\right|_{\lambda_{0}=\hat{\lambda}_{0}}=0 ;$ then, given $(\hat{\beta}, \hat{v})$, new estimates $\hat{\alpha}$ are obtained by solving $\partial p_{\beta, v}\left(h_{p}\right) / \partial \alpha=0$.

Step 3: Repeat Step 2 until the maximum absolute difference between the previous and current estimates for $(\beta, v)$ and $\alpha$ is less than $10^{-6}$.

After convergence, we compute the estimates of the standard errors of $\hat{\beta}$ and $\hat{\alpha}$.

\section{Illustration: kidney infection data}

To demonstrate differences of various estimation methods in small cluster size $n_{i} \equiv 2$, we use the kidney infection data (McGilchrist and Aisbett, 1991). The data consist of times until the first and second recurrences $\left(n_{i} \equiv 2\right)$ of kidney infection in $38(q=38)$ patients using a portable dialysis machine. Each survival time (time) is the time until infection since the insertion of the catheter. The survival times for the same patient are likely to be related because of a shared frailty describing the common patient's effect. The catheter is later removed if infection occurs and can be removed for other reasons, which we regard as censoring; about $24 \%$ of the data were censored.

We fit frailty models with two covariates, the sex ( 1 = male; 2 = female) and age of each patient, using the functions (frailtyHL(), coxph(), coxme() and phmm()) in the four packages. The results are summarized in Table 2.

Table 2: Comparison of different estimation methods for the kidney infection data

\begin{tabular}{c|ccc}
\hline & Sex & Age & $\begin{array}{c}\text { Patient } \\
\text { Method }\end{array}$ \\
$\hat{\beta}_{1}(\mathrm{SE})$ & $\hat{\beta}_{2}(\mathrm{SE})$ & $\hat{\alpha}(\mathrm{SE})$ \\
\hline lognormal model & & & \\
HL(0,1) & -1.380 & 0.005 & 0.535 \\
& $(0.431)$ & $(0.012)$ & $(0.338)$ \\
HL(1,1) & -1.414 & 0.005 & 0.545 \\
& $(0.432)$ & $(0.012)$ & $(0.340)$ \\
coxph() & -1.388 & 0.005 & 0.551 \\
(Breslow) & $(0.441)$ & $(0.012)$ & $(-)$ \\
coxph() & -1.411 & 0.005 & 0.569 \\
(Efron) & $(0.445)$ & $(0.013)$ & $(-)$ \\
coxme() & -1.332 & 0.005 & 0.440 \\
(Breslow) & $(0.414)$ & $(0.012)$ & $(-)$ \\
coxme() & -1.355 & 0.004 & 0.456 \\
(Efron) & $(0.417)$ & $(0.012)$ & $(-)$ \\
phmm() & -1.329 & 0.004 & 0.378 \\
& $(0.452)$ & $(0.012)$ & $(-)$ \\
\hline gamma model & & & \\
HL(0,2) & -1.691 & 0.007 & 0.561 \\
& $(0.483)$ & $(0.013)$ & $(0.280)$ \\
HL(1,2) & -1.730 & 0.007 & 0.570 \\
& $(0.485)$ & $(0.013)$ & $(0.281)$ \\
coxph () & -1.557 & 0.005 & 0.398 \\
(Breslow) & $(0.456)$ & $(0.012)$ & $(-)$ \\
coxph() & -1.587 & 0.005 & 0.412 \\
(Efron) & $(0.461)$ & $(0.012)$ & $(-)$ \\
\hline
\end{tabular}

In PPL procedures (coxph () and coxme ()), the Breslow method provides slightly smaller estimate for $\alpha$ than the Efron method. In the lognormal frailty, REMPL procedures (fraityHL() and coxph ()) give larger estimates for $\alpha$ than ML (phmm ()) and MPL (coxme ()) procedures. However, both ML and MPL estimates from phmm() and coxme() are somewhat 


\section{$[1] \quad 335.97 \quad 397.36 \quad 362.14 \quad$, CAIC mAIC rAIC \\ $[1] \quad 362.22 \quad 366.14 \quad$,}

The code and results for the gamma frailty model with HL(1,2) are presented below. The output shows that these results are similar to those of the lognormal frailty, particularly for estimation of $\beta$. The deviance difference (based on REMPL) between the Cox model and gamma frailty model using the second-order approximation $-2 s_{\beta, v}\left(h_{p}\right)$ is $364.15-$ $362.12=2.03(<2.71)$, again indicating the absence of frailty effect (i.e. $\alpha=0$ ) as evident in the lognormal frailty model.

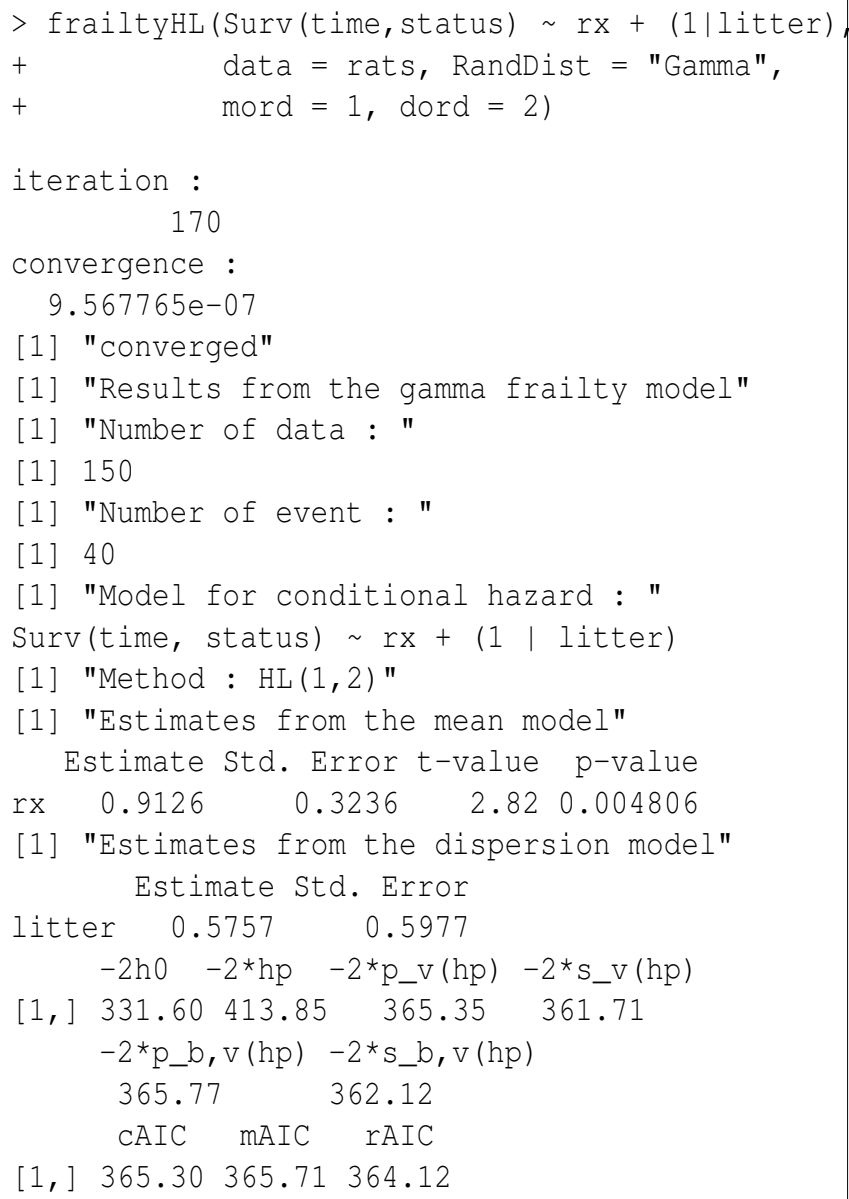

For the selection of a model among nested or non-nested models such as lognormal and gamma frailty models, we may use three Akaike information criteria (AIC) (Lee et al., 2006; Donohue et al., 2011; Ha et al., 2007) based on conditional likelihood, marginal likelihood and restricted likelihood, respectively, defined by

$$
\begin{aligned}
\mathrm{cAIC} & =-2 h_{0}+2 \mathrm{df}_{\mathrm{c}}, \\
\mathrm{mAIC} & =-2 p_{v}\left(h_{p}\right)+2 \mathrm{df}_{\mathrm{m}}, \\
\mathrm{rAIC} & =-2 p_{\beta, v}\left(h_{p}\right)+2 \mathrm{df}_{\mathrm{r}},
\end{aligned}
$$

where $h_{0}=\ell_{0}^{*}$ in $(4)$, and

$$
\mathrm{df}_{\mathrm{c}}=\operatorname{trace}\left\{D^{-1}\left(h_{p},(\beta, v)\right) D\left(h_{0},(\beta, v)\right)\right\}
$$

is an 'effective degrees of freedom adjustment' for estimating the fixed and random effects, computed using the Hessian matrices $D\left(h_{p},(\beta, v)\right)=$ $-\partial^{2} h_{p} / \partial(\beta, v)^{2}$ and $D\left(h_{0},(\beta, v)\right)=-\partial^{2} h_{0} / \partial(\beta, v)^{2}$. Note here that $\mathrm{df}_{\mathrm{m}}$ is the number of fixed parameters and $\mathrm{df}_{\mathrm{r}}$ is the number of dispersion parameters (Ha et al., 2007). For calculation of the mAIC and rAIC of gamma frailty model using $\operatorname{HL}(0,2)$ or $\mathrm{HL}(1,2)$, we use the corresponding second-order approximations, defined by $\mathrm{mAIC}=-2 s_{v}\left(h_{p}\right)+2 \mathrm{df}_{\mathrm{m}}$ and $\mathrm{rAIC}=-2 s_{\beta, v}\left(h_{p}\right)+2 \mathrm{df}_{\mathrm{r}}$. We select a model to minimize the AIC values among models. If the AIC difference is larger than 1 the choice can be made (Sakamoto et al., 1986). However, if the difference is less than 1 a simpler model can be selected by a parsimony principle (Donohue et al., 2011).

In the data set, in the Cox model $\mathrm{cAIC}=365.69$, $\mathrm{mAIC}=365.69$ and $\mathrm{rAIC}=364.15$, and in the lognormal frailty model $\mathrm{cAIC}=362.22$, $\mathrm{mAIC}=366.14$ and $\mathrm{rAIC}=364.56$, and in the gamma frailty model $\mathrm{cAIC}=365.30, \mathrm{mAIC}=365.71$ and $\mathrm{rAIC}=364.12$. The likelihood tests based upon the REMPL showed the absence of frailty effect $(\alpha=0)$, so that MAIC and rAIC of all the three models are similar. Thus, we may choose the parsimonious Cox model. However, the cAIC selects the lognormal model, indicating that this model could give better prediction.

\section{Example 2: Multilevel frailty models on CGD infection data}

The CGD data set presented by Fleming and Harrington (1991) consists of a placebo-controlled randomized trial of gamma interferon (rIFN-g) in the treatment of chronic granulomatous disease (CGD). 128 patients $(i d)$ from 13 centers $\left(q_{1}=13, q_{2}=128\right)$ were tracked for around 1 year. The number (i.e. cluster size) of patients per center ranged from 4 to 26. The survival times (tstop-tstart) are the recurrent infection times of each patient from the different centers. Censoring occurred at the last observation for all patients, except one, who experienced a serious infection on the day he left the study; in the CGD study about $63 \%$ of the data were censored. The recurrent infection times for a given patient are likely to be correlated. However, each patient belongs to one of the 13 centers; hence, the correlation may also be attributed to a center effect.

Ignoring important random components may render invalid many of the traditional statistical analysis techniques. We fit a multilevel lognormal frailty model with two frailties and a single covariate, treat (rIFN-g, placebo), using frailtyHL (). Here, the two frailties are random center and patient effects. The code and results using $\mathrm{HL}(1,1)$ are provided below. The output shows that the effect of treatment is significant ( $\mathrm{t}$-value $=-3.476$ with $\mathrm{p}$-value $<0.001$ ), indicating that rIFN-g significantly reduces the rate of serious infection in CGD patients. The estimate of 
variance of patient frailty $\left(\hat{\alpha}_{2}=1.002\right)$ is considerably larger than variance of center frailty $\left(\hat{\alpha}_{1}=0.030\right)$, indicating that the random-patient effect is more heterogeneous.

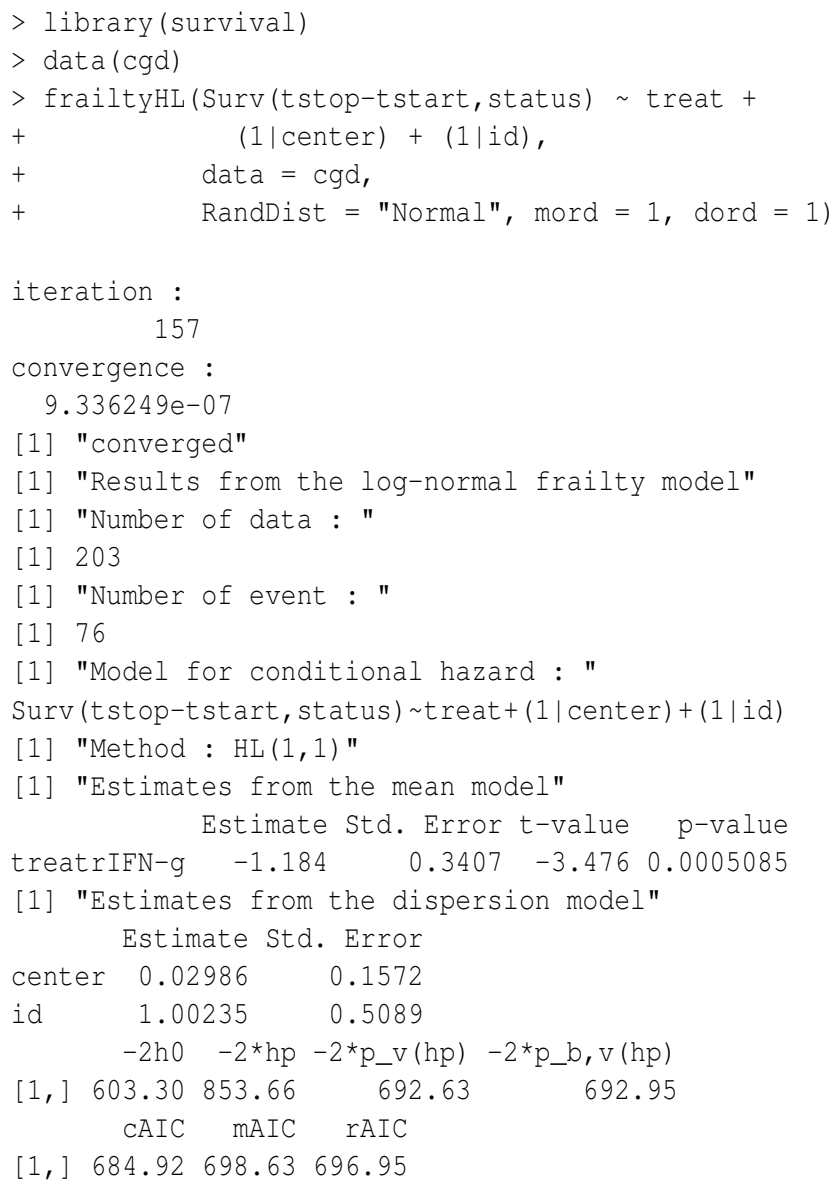

For testing the absence of a random component $\left(\alpha_{1}=0\right.$ or $\left.\alpha_{2}=0\right)$, we use the deviance based on REMPL, $-2 p_{\beta, v}\left(h_{p}\right)$, and fit the following four models including the Cox model and three lognormal frailty models using HL(1,1) method,

M1: Cox model without frailty $\left(\alpha_{1}=0, \alpha_{2}=0\right)$ has $-2 p_{\beta, v}\left(h_{p}\right)=707.48$,

M2: model without patient effect $\left(\alpha_{1}>0, \alpha_{2}=0\right)$ has $-2 p_{\beta, v}\left(h_{p}\right)=703.66$,

M3: model without center effect $\left(\alpha_{1}=0, \alpha_{2}>0\right)$ has $-2 p_{\beta, v}\left(h_{p}\right)=692.99$, and

M4: multilevel model above requiring both patient and center effects $\left(\alpha_{1}>0, \alpha_{2}>0\right)$ has $-2 p_{\beta, v}\left(h_{p}\right)=692.95$.

The deviance difference between M3 and M4 is $692.99-692.95=0.04$, which is not significant at a $5 \%$ level $\left(\chi_{1,0.10}^{2}=2.71\right)$, indicating the absence of the random-center effects, i.e. $\alpha_{1}=0$. The deviance difference between M2 and M4 is 703.66-692.95=10.71, indicating that the random-patient effects are necessary, i.e. $\alpha_{2}>0$. In addition, the deviance difference between M1 and M3 is 707.48-692.99=14.49, indicating that the random-patient effects are indeed necessary with or without random-center effects.

Let us choose a final model using information criteria. For $\mathrm{M} 1$ we have $\mathrm{cAIC}=708.68, \mathrm{mAIC}=708.68$ and $\mathrm{rAIC}=707.48$; for $\mathrm{M} 2 \mathrm{cAIC}=702.96, \mathrm{mAIC}=706.88$ and $\mathrm{rAIC}=705.66$; for $\mathrm{M} 3 \mathrm{cAIC}=684.84, \mathrm{mAIC}=696.68$ and $\mathrm{rAIC}=694.99$; for $\mathrm{M} 4 \mathrm{cAIC}=684.92, \mathrm{mAIC}=698.63$ and $\mathrm{rAIC}=696.95$. All of the three criteria choose M3 in the CGD data set.

\section{Comparison of results with alternative procedures}

Using the examples in the previous section, we compare the outcomes from frailtyHL and other packages. We consider the three functions ( coxph (), coxme () and phmm ()) for the lognormal frailty model and the coxph() function for the gamma frailty model.

\section{Example 1: Rat data}

The codes of coxph (), coxme () and phmm () for fitting lognormal frailty model are, respectively, as follows:

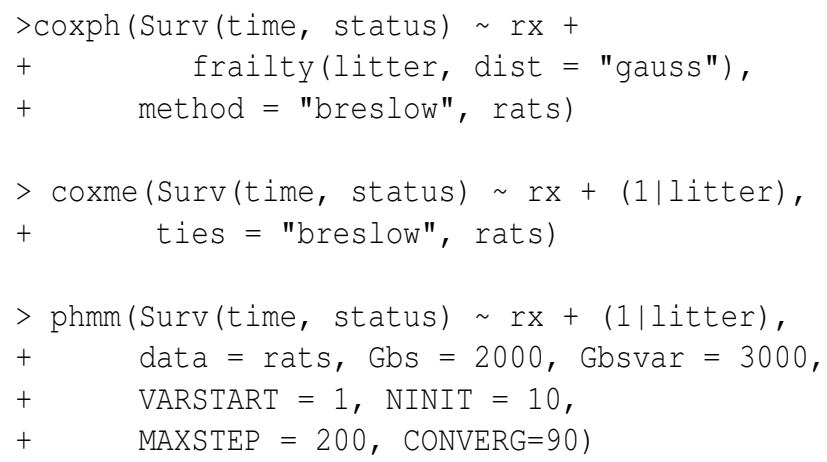

Table 3 summarizes the estimation results. Even though cluster size $n_{i} \equiv 3$ is not large, the results are similar. For example, MPL and ML estimates for $\alpha$ from coxme () and phmm () are somewhat different in Table 2 when $n_{i} \equiv 2$, while they become similar in Table 3.

Next, the code of coxph () for fitting the gamma frailty model is below:

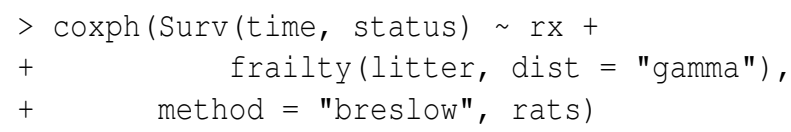

The results of frailtyHL() $(\mathrm{HL}(0,2), \mathrm{HL}(1,2))$ and coxph ( ) for gamma frailty are also presented in Table 3 . For the estimation of $\beta$ both results from frailtyHL () and coxph () are similar, but for $\alpha$ they are somewhat different. That is, our REMPL estimates from frailtyHL ( ) $(\hat{\alpha}=0.575$ with $\operatorname{HL}(0,2)$ and $\hat{\alpha}=0.576$ with $\operatorname{HL}(1,2))$ are somewhat larger than the ML estimates from coxph ( ) $(\hat{\alpha}=0.474$ with Breslow method and $\hat{\alpha}=0.499$ with Efron method). 
Table 3: Comparison of different estimation methods for the rat data

\begin{tabular}{c|ll}
\hline Method & $\begin{array}{l}\text { Rx } \\
\hat{\beta}(\mathrm{SE})\end{array}$ & $\begin{array}{l}\text { Litter } \\
\hat{\alpha}(\mathrm{SE})\end{array}$ \\
\hline $\begin{array}{c}\text { lognormal model } \\
\text { HL(0,1) }\end{array}$ & $0.906(0.323)$ & $0.427(0.423)$ \\
HL(1,1) & $0.911(0.323)$ & $0.427(0.423)$ \\
$\begin{array}{c}\text { coxph () } \\
\text { (Breslow) } \\
\text { coxph() } \\
\text { (Efron) }\end{array}$ & $0.905(0.322)$ & $0.395(-)$ \\
$\begin{array}{c}\text { coxme () } \\
\text { (Breslow) } \\
\text { coxme () } \\
\text { (Efron) } \\
\text { phmm() }\end{array}$ & $0.913(0.323)$ & $0.412(-)$ \\
\hline $\begin{array}{c}\text { gamma model } \\
\text { HL(0,2) }\end{array}$ & $0.905(0.322)$ & $0.406(-)$ \\
$\begin{array}{c}\text { HL(1,2) } \\
\text { coxph () }\end{array}$ & $0.908(0.324)$ & $0.575(0.598)$ \\
$\begin{array}{c}\text { (Efron) } \\
\text { coxph () }\end{array}$ & $0.913(0.324)$ & $0.576(0.598)$ \\
\hline
\end{tabular}

\section{Example 2: CGD data}

The code of the coxme () function for fitting multilevel lognormal frailty model is as follows:

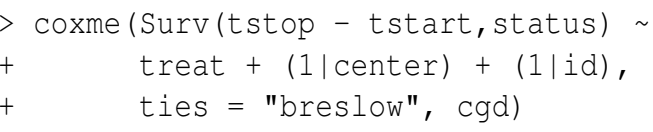

The results of frailtyHL() $(\mathrm{HL}(0,1), \mathrm{HL}(1,1))$ and coxme () are summarized in Table 4 . The results from HL and PPL methods for frailty parameters become more similar because the cluster sizes (the number of patients from different centers) are somewhat large, ranging from 4 to 26 .

Table 4: Comparison of different estimation methods for the CGD data

\begin{tabular}{c|ccc}
\hline & $\begin{array}{c}\text { Treat } \\
\text { Method }\end{array}$ & $\begin{array}{c}\text { Center } \\
\hat{\beta}(\mathrm{SE})\end{array}$ & $\begin{array}{c}\text { Patient } \\
\hat{\alpha}_{1}(\mathrm{SE})\end{array}$ \\
$\hat{\alpha}_{2}(\mathrm{SE})$ \\
\hline lognormal model & & & \\
HL(0,1) & -1.074 & 0.026 & 0.982 \\
& $(0.335)$ & $(0.153)$ & $(0.501)$ \\
HL(1,1) & -1.184 & 0.030 & 1.002 \\
& $(0.341)$ & $(0.157)$ & $(0.509)$ \\
coxme () & -1.074 & 0.033 & 0.939 \\
(Breslow) & $(0.333)$ & $(-)$ & $(-)$ \\
coxme () & -1.074 & 0.032 & 0.947 \\
(Efron) & $(0.333)$ & $(-)$ & $(-)$ \\
\hline
\end{tabular}

\section{Summary}

The h-likelihood method offers novel possibilities to fit various models with random effects. The frailtyHL package for frailty models eliminates the nuisance parameters $\lambda_{0}$ in the h-likelihood (3) by profiling. Such models have important applications in multi-center clinical study (Vaida and $\mathrm{Xu}, 2000$ ), meta analysis (Rondeau et al., 2008), and genetic analysis (Lee et al., 2006). Therefore, this package can be potentially adopted by statisticians in several different fields.

\section{Acknowledgements}

This research was supported by Basic Science Research Program through the National Research Foundation of Korea (NRF) funded by the Ministry of Education, Science and Technology (No. 20090088978, No. 2010-0021165 and No. 2010-0011372).

\section{Bibliography}

M. Alam, L. Ronnegard, and X. Shen. hglm: hierarchical generalized linear models, 2010. URL http: / /CRAN.R-project.org/package=hglm. R package version 1.1.1. [p28]

N. E. Breslow. Discussion of Professor Cox's paper. Journal of the Royal Statistical Society Series B, 34: 216-217, 1972. [p29]

N. E. Breslow. Covariance analysis of censored survival data. Biometrics, 30:89-99, 1974. [p30]

D. R. Cox and N. Reid. Parameter orthogonality and approximate conditional inference (with discussion). Journal of the Royal Statistical Society Series $B, 49: 1-39,1987$. [p30]

L. Duchateau and P. Janssen. The Frailty Model. Springer-Verlag: New York, 2008. [p28]

M. Donohue, R. Overholser, R. Xu, and F. Vaida. Conditional Akaike information under generalized linear and proportional hazards mixed models. Biometrika, 98:685-700, 2011. [p33]

M. Donohue and R. Xu. phmm: proportional hazards mixed-effects model, 2012. URL http: //CRAN. $\mathrm{R}$-project.org/package $=$ phmm. R package version $0.7-4$. [p28, 30, 31]

T. R. Fleming and D. P. Harrington. Counting Processes and Survival Analysis. Wiley: New York, 1991. [p29, 33]

J. R. Gonzalez, V. Rondeau Y. Mazroui, A. Mauguen, and A. Diakité. frailtypack: frailty models using a semi-parametrical penalized likelihood estimation or a 
parametrical estimation, 2012. URL http://CRAN. $\mathrm{R}$-project.org/package=frailtypack. R package version 2.2-23. [p28]

I. D. Ha and Y. Lee. Estimating frailty models via Poisson hierarchical generalized linear models. Journal of Computational and Graphical Statistics, 12: 663-681, 2003. [p28, 30]

I. D. Ha and Y. Lee. Comparison of hierarchical likelihood versus orthodox best linear unbiased predictor approaches for frailty models. Biometrika, 92: 717-723, 2005. [p28, 30]

I. D. Ha, Y. Lee and G. MacKenzie. Model selection for multi-component frailty models. Statistics in Medicine, 26:4790-4807, 2007. [p29, 33]

I. D. Ha, Y. Lee and J. K. Song. Hierarchical likelihood approach for frailty models. Biometrika, 88: 233-243, 2001. [p28, 29, 30]

I. D. Ha, M. Noh and Y. Lee. Bias reduction of likelihood estimators in semi-parametric frailty models. Scandinavian Journal of Statistics, 37:307-320, 2010. [p30, 31, 32]

I. D. Ha, M. Noh and Y. Lee. frailtyHL: frailty models using h-likelihood, 2012. URL http://CRAN. $\mathrm{R}$-project.org/package=frailtyHL. R package version 1.1. [p28, 30]

I. D. Ha, T. Park and Y. Lee. Joint modelling of repeated measures and survival time data. Biometrical Journal, 45:647-658, 2003. [p32]

I. D. Ha, R. Sylvester, C. Legrand and G. MacKenzie. Frailty modelling for survival data from multicentre clinical trials. Statistics in Medicine, 30:21442159, 2011. [p31, 32]

P. Hougaard. Analysis of Multivariate Survival Data. Springer-Verlag: New York, 2000. [p28, 29]

Y. Lee and I. D. Ha. Orthodox BLUP versus hlikelihood methods for inferences about random effects in Tweedie mixed models. Statistics and Computing, 20:295-303, 2010. [p31]

Y. Lee, M. Jang and W. Lee. Prediction interval for disease mapping using hierarchical likelihood. Computational Statistics, 26:159-179, 2011. [p31]

Y. Lee and J. A. Nelder. Hierarchical generalized linear models (with discussion). Journal of the Royal Statistical Society Series B, 58:619-678, 1996. [p28, 30]

Y. Lee and J. A. Nelder. Hierarchical generalised linear models: a synthesis of generalised linear models, random-effect models and structured dispersions. Biometrika, 88:987-1006, 2001. [p30]
Y. Lee and J. A. Nelder. Double hierarchical generalised linear models (with discussion). Applied Statistics, 55:139-185, 2006. [p32]

Y. Lee, J. A. Nelder and Y. Pawitan. Generalised Linear Models with Random Effects: Unified Analysis via Hlikelihood. Chapman \& Hall: London, 2006. [p28, $30,31,32,33,35]$

N. Mantel, N. R. Bohidar and J. L. Ciminera. Mantel-Haenszel analyses of litter-matched timeto-response data, with modifications for recovery of interlitter information. Cancer Research, 37:38633868, 1977. [p32]

C. A. McGilchrist and C. W. Aisbett. Regression with frailty in survival analysis. Biometrics, 47:461-466, 1991. [p31]

M. Molas. HGLMMM: hierarchical generalized linear models, 2010. URL http://CRAN.R-project.org/ package=HGLMMM. R package version 0.1.1. [p28]

M. Noh and Y. Lee. dhglm: double hierarchical generalized linear models, 2011. URL http://CRAN. $\mathrm{R}$-project.org/package=dhglm. R package version 1.0. [p28]

S. Ripatti and J. Palmgren. Estimation of multivariate frailty models using penalized partial likelihood. Biometrics, 56:1016-1022, 2000. [p30]

V. Rondeau, S. Michiels, B. Liquet and J. P. Pignon. Investigating trial and treatment heterogeneity in an individual patient data meta-analysis of survival data by means of the penalized maximum likelihood approach. Statistics in Medicine, 27:1894910, 2008. [p35]

Y. Sakamoto, M. Ishiguro and G. Kitagawa. Akaike Information Criterion Statistics. KTK Scientific Publisher: Tokyo, 1986. [p33]

T. M. Therneau. survival: survival analysis, including penalised likelihood, 2010. URL http:// CRAN.R-project.org/package=survival. R package version 2.36-2. [p28, 30, 31]

T. M. Therneau. coxme: Mixed Effects Cox Models, 2011. URL http: / / CRAN. R-project . org/package $=$ coxme. R package version 2.2-1. [p28, 30]

T. M. Therneau and P. M. Grambsch. Modelling Survival Data: Extending the Cox Model. SpringerVerlag: New York, 2000. [p29, 30, 31]

F. Vaida and R. Xu. Proportional hazards model with random effects. Statistics in Medicine, 19:3309-3324, 2000. [p32, 35]

K. K. W. Yau. Multilevel models for survival analysis with random effects. Biometrics, 57:96-102, 2001. [p29] 
Il Do Ha

Department of Asset Management

Daegu Haany University, Korea

idha@dhu.ac.kr

Maengseok Noh

Department of Statistics
Pukyong National University, Korea

msnohepknu.ac.kr

Youngjo Lee

Department of Statistics

Seoul National University, Korea

young jodsnu.ac.kr 